\title{
Factores críticos de éxito para las empresas distribuidoras de insumos médicos
}

\author{
Critical success factors for companies that distribute medical supplies
}

Fatores críticos de sucesso para empresas de distribuição de suprimentos médicos

Recibido: octubre 2019

Arbitrado: noviembre 2019

Aceptado: diciembre 2019

Publicado: enero 2021 $\triangle$ Mariana Caballero Salinas

mari24_296@hotmail.com

https://orcid.org/0000-0003-3397-8737

Universidad Andina Simón Bolívar, Sucre-Bolivia

\section{RESUMEN}

Este estudio busca determinar los factores críticos de éxito sobre la competitividad de las empresas del sector de insumos médicos en la ciudad de Sucre, Bolivia, mediante el desarrollo de un censo aplicado a 25 empresas que conforman el sector. Empleando la metodología de Quinn y Rorbaug, se indagó acerca de los factores críticos partiendo de 3 dimensiones (enfoque de la organización, estructura organizacional, medios organizacionales) y las variables que determinan competitividad empresarial a partir de 4 modelos (procesos internos, sistema abierto, racional, relaciones humanas). Un estudio estadístico univariado permitió seleccionar 6 factores críticos de éxito (posición tecnológica, calidad de productos, innovación de productos, innovación en procesos, formación del personal y capacidad directiva). Consecuentemente se validó cada factor por un análisis multivariante, descartando 3 y comprobando que existen solamente 3 factores críticos de éxito que garantizan la competitividad en el sector: capacidad directiva, innovación en procesos y posición tecnológica.

Palabras clave: Gestión empresarial; competitividad; factores de riesgo
This study seeks to determine the critical success factors on the competitiveness of companies in the medical supplies sector in the city of Sucre, Bolivia, through the development of a census applied to 25 companies that make up the sector. Using the Quinn and Rorbaug methodology, the critical factors were investigated based on 3 dimensions (organizational approach, organizational structure, organizational means) and the variables that determine business competitiveness from 4 models (internal processes, open system, rational, human relations). A univariate statistical study made it possible to select 6 critical success factors (technological position, product quality, product innovation, process innovation, personnel training, and managerial capacity). Consequently, each factor was validated by a multivariate analysis, discarding 3 and verifying that there are only 3 critical success factors that guarantee competitiveness in the sector: managerial capacity, process innovation and technological position.

Key words: Business management; competitiveness; risk factor's

\section{RESUMO}

Este estudo busca determinar os fatores críticos de sucesso na competitividade das empresas do setor de suprimentos médicos da cidade de Sucre, Bolívia, por meio do desenvolvimento de um censo aplicado a 25 empresas que compõem o setor. Utilizando a metodologia Quinn e Rorbaug, os fatores críticos foram investigados a partir de 3 dimensões (abordagem organizacional, estrutura organizacional, meios organizacionais) e as variáveis que determinam a competitividade empresarial a partir de 4 modelos (processos internos, sistema aberto, racional, relações humanas). Um estudo estatístico univariado permitiu selecionar 6 fatores críticos de sucesso (posição tecnológica, qualidade do produto, inovação do produto, inovação do processo, treinamento do pessoal e capacidade gerencial). Consequentemente, cada fator foi validado por uma análise multivariada, descartando 3 e verificando que existem apenas 3 fatores críticos de sucesso que garantem a competitividade do setor: capacidade gerencial, inovação de processos e posição tecnológica.

Palavras-chave: Gestão de negócios; competitividade; Fatores de risco 


\section{INTRODUCCIÓN}

A nte un entorno empresarial cada vez más cambiante, complejo y dinámico, se ha vuelto esencial estudiar aquellos elementos claves que permiten que una empresa pueda ser competitiva en el mercado donde se desenvuelve. Estos elementos clave o factores críticos de éxito son eventos, condiciones, circunstancias o actividades que de tener resultados óptimos garantizan la alta competitividad de la empresa, Villegas (2005).

A nivel mundial, son cada vez más empresas las que reconocen la necesidad de identificar estos factores clave, ya que resulta crucial en el éxito o fracaso institucional. Sin embargo, en su mayoría, las empresas distribuidoras de insumos médicos no se adecuan a las características de su mercado. Al no haber una correcta identificación de los factores de éxito en este tipo de empresas, se incurre en el error de adoptar medidas poco estratégicas tanto en su venta, como en la importación de mercadería, PROCHILE (2016).

Esta necesidad no escapa al mercado de insumos médicos en la ciudad de Sucre, Bolivia, que lejos de parecer un mercado comercial típico, presenta particularidades propias que si no son analizadas y aprovechadas se convierten en un obstáculo para la competitividad de la empresa, PROCHILE (2016). Sin embargo, en la actualizad sólo algunas empresas del sector destinan esfuerzos a investigar cuales son los factores específicos que las llevan al éxito o fracaso, y las pocas que estudian los factores se limitan únicamente al manejo de inventarios, por tanto, dejan de lado otros procesos como ser compras, ventas en licitaciones, entre otros.

El estudio de los factores críticos tiene características particulares a tomar en cuenta que el mercado de insumos y medicamentos en Sucre, debido a que se torna complejo al referirse a los participantes, pues integra elementos particulares: en primer lugar, la participación del Estado como ofertante a través de producciones nacionales y demandante por medio de instituciones de salud pública; en segundo lugar, la participación de empresas privadas que también actúan como ofertantes a través de distribuidoras de insumos médicos y demandantes por medio de otras instituciones de salud.

Así, las empresas de insumos médicos manejan grandes niveles de inversión, por tanto, el riesgo que asumen los inversionistas de las empresas es elevado, así como el nivel de rentabilidad que esperan obtener por tal riesgo, a pesar de ello, no se orientan adecuadamente las estrategias de las empresas y si bien algunas empresas logran obtener niveles elevados de rentabilidad, otras no están logrando conseguir altos márgenes de ganancia.

Por lo expuesto, el estudio de los factores críticos que intervienen en el éxito de una empresa distribuidora de insumos médicos frente a su competencia se vuelve trascendental, pues considerando que este mercado presenta elementos clave bastante diferentes de cualquier otro modelo propuesto hasta ahora, corresponde un estudio que les permita gestionar sus procesos y orientar sus esfuerzos eficientemente.

Por tanto, este estudio aporta una respuesta científica al problema de cómo incrementar la competitividad de las empresas distribuidoras de insumos médicos en el mercado de Sucre, considerando sus factores críticos de éxito. Dado que estos factores que pueden impulsar 
o frenar el avance de las organizaciones, una correcta integración de dichos factores se convierte en una ventaja competitiva de la empresa en el mercado, Villegas (2005).

Corresponde hacer referencia a tres estudios relacionados al análisis de factores de éxito competitivo desarrollados en Colombia y España el 2005, en Venezuela y México el 2009. En estos estudios se empleó la metodología propuesta por Quinn y Rourgbagh. De esta manera, Villegas (2005), manifiesta la elaboración de indicadores de gestión como instrumento de medida de los factores críticos de éxito. Conforme a Estrada (2009), en su estudio empírico se establece una variedad de factores críticos productos de los análisis, luego en su posterior clasificación contrasta cada una de las variables a través de modelos de regresión estadísticos. Más adelante fue también Aragón (2005), quien estableció la posibilidad de incorporar análisis univariantes dentro del análisis para enriquecer el efecto de cada factor en el modelo de regresión final.

Respecto a la medición del desempeño de un individuo, estos indicadores miden y controlan el progreso a corto plazo del individuo en la ejecución de tareas que han sido asignadas para el logro de una dimensión crítica (factor crítico de éxito). Otra forma de visualizarlo es midiendo el progreso a corto plazo hacia los objetivos a largo plazo.

Con relación a la variable competitividad, en 1983, Quinn y Rorbaugh establecieron cuatro modelos desde los cuales se puede analizar la competitividad de las empresas de forma cualitativa y expresarlo finalmente de forma cuantitativa. La ventaja del modelo de Quinn radica en su visión multidimensional de lo que es competitividad para poder ser analizado desde diferentes perspectivas. Según Romero (2009), la formulación de los indicadores de competencia en los cuatro modelos de Quinn y Rorbaugh resultan de la media aritmética de los 3 ítems por cada modelo, de esta forma se formularon los 4 indicadores correspondientes a los cuatro modelos, incluyendo además un resultado global, resultado de la media aritmética de los 12 ítems.

Después de la identificación de los factores críticos en una empresa u organización, resulta importante conocer su clasificación, a fin de establecer una correcta integración de estos. Según King (2006), existen 7 factores críticos de éxito: soporte de alta gerencia, equipo competitivo, cooperación interdepartamental, objetivos y metas claras, gestión del proyecto, comunicación interdepartamental y selección de empaques. Por su parte Mendoza (2007), señala 9 factores críticos de éxito: Compromiso de la dirección, creación de equipos multidisciplinarios, definición de objetivos, integración interdepartamental, comunicación de la estrategia, compromiso del personal, manejo de información del cliente, servicio al cliente y automatización del marketing.

Con un enfoque dirigido a Pymes, Aragón (2005), postula 6 factores a tomar en cuenta: tamaño de la empresa, estructura poco jerarquizada, innovación en productos, innovación en procesos, posición tecnológica y técnicas económicas financieras. De la misma manera, Estrada (2009) también estudia los factores críticos de éxito para Pymes y enuncia 6: planeación estratégica, innovación en productos, innovación en procesos, certificación de calidad, posición tecnológica y recursos Humanos. 
Como se puede observar, son diversos los factores que se pueden valorar, es pertinente afirmar que deberán ser analizados en cada contexto particular tomando en cuenta las condiciones de la empresa, del mercado y del sector.

\section{MÉTODO}

e trató de un estudio de enfoque mixto y alcance relacional. La primera etapa, el componente cualitativo, consistió en el desarrollo de un diagnóstico de las empresas objeto de estudio. Para esto se aplicó la técnica de la observación y entrevistas a los principales involucrados y tomadores de decisiones. Con base en la información recogida se aplicaron las herramientas Diamante de Porter, análisis PESTEL y análisis de la Empresa Líder, a continuación, se presentó un cuadro resumen de los 3 análisis.

La segunda etapa, el componente cuantitativo, consistió en la ejecución de un censo aplicado a las 25 empresas, compuesto por dos cuestionarios de preguntas cerradas. El primer cuestionario estuvo conformado por 10 preguntas en escala Likert referidas a la compresión y percepción los factores críticos de éxito, este instrumento fue validado gracias al diagnóstico anteriormente desarrollado (Diamante de Porter, análisis PESTEL y análisis de la Empresa Líder), y otro cuestionario previamente validado por Estrada (2009), en escala Likert con 9 preguntas enfocadas a medir la competitividad de las empresas.

Para el análisis de los factores críticos de éxito se tomó tres ejes o dimensiones. El primero relacionado con el enfoque de la organización, que va desde un punto de vista interno (basado en una visión micro sobre el buen entendimiento y el desarrollo del personal) a uno externo (énfasis a nivel macro del éxito de la empresa). El segundo se centró en la estructura organizacional e hizo hincapié en la estabilidad y la flexibilidad de la empresa. Por último, el tercero se relacionó con los medios y fines organizacionales.

Para medir la competitividad se tomó cuatro modelos, para valorar los distintos modelos se utilizaron 12 ítems (tres por cada modelo) con una escala tipo Likert de 1 a 4.

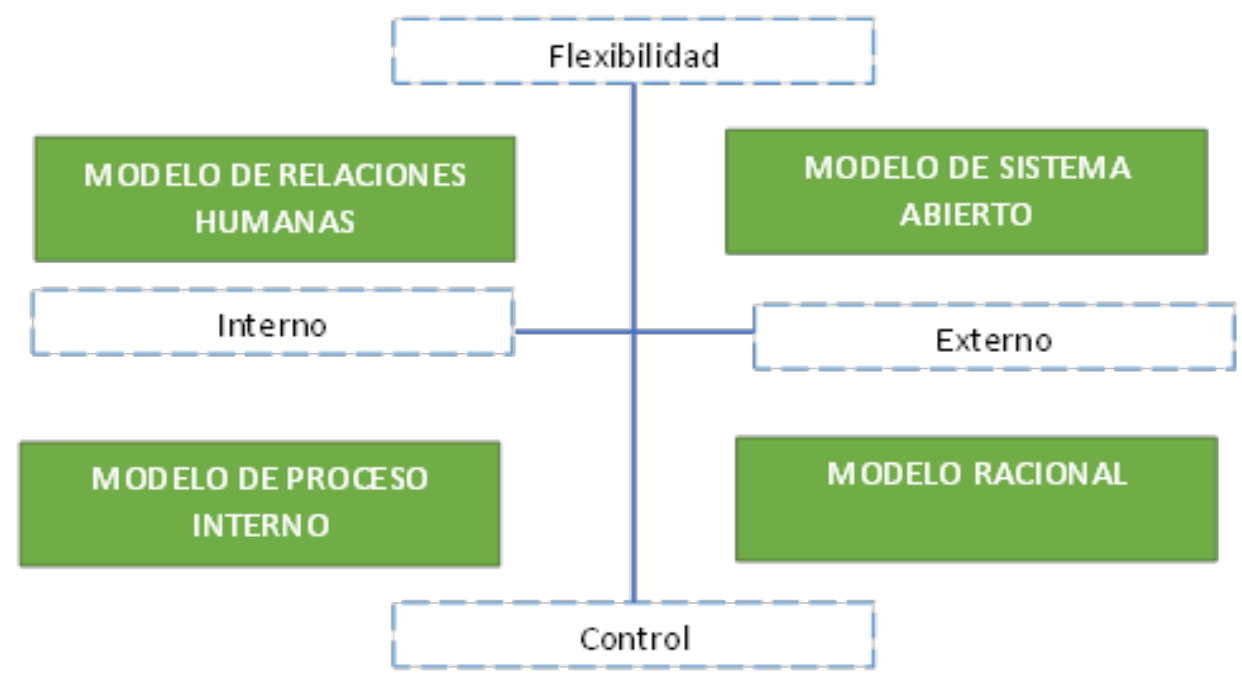

Gráfico 1. Modelo de Quinn y Rorbaugh. 
La variable que representó cada modelo se construyó a partir de la media aritmética de los tres ítems, con lo cual resultó un rango teórico de 1 a 4. Para validar estas medidas se verificó la fiabilidad de las escalas a través del estadístico Alpha de Cronbach, Los valores van desde 0 a 1 . Los valores de 0.60 hasta 0.70 fueron considerados el límite inferior de aceptabilidad. En la tabla $N^{\circ} 1$ se expone el detalle de los ítems utilizados, así como los criterios de la validación de las escalas para cada modelo de rendimiento.

Tabla 1. Esquema de ítems de valoración para el modelo.

\begin{tabular}{|c|c|c|}
\hline Dimensión & Item & Indicador \\
\hline $\begin{array}{l}\text { Modelo de } \\
\text { Procesos } \\
\text { Internos }\end{array}$ & $\begin{array}{l}\text { Mejora en la calidad del producto. } \\
\text { Mejora en la coordinación de procesos internos. } \\
\text { Mejor organización de las tareas del personal }\end{array}$ & $\begin{array}{l}\text { Valoración de la evolución de } \\
\text { los aspectos en los últimos } \\
2 \text { años }(1=\text { situación muy } \\
\text { desfavorable, } 4=\text { situación } \\
\text { muy favorable) }\end{array}$ \\
\hline $\begin{array}{l}\text { Modelo de } \\
\text { Sistema } \\
\text { Abierto }\end{array}$ & $\begin{array}{l}\text { Aumento de la satisfacción de los clientes. } \\
\text { Incremento de la habilidad de adaptación } \\
\text { a las necesidades de los mercados. } \\
\text { Mejora de la imagen de empresa y de sus productos. }\end{array}$ & $\begin{array}{l}\text { Valoración de la evolución de } \\
\text { los aspectos en los últimos } \\
2 \text { años }(1=\text { situación muy } \\
\text { desfavorable, } 4=\text { situación } \\
\text { muy favorable) }\end{array}$ \\
\hline $\begin{array}{l}\text { Modelo } \\
\text { Racional }\end{array}$ & $\begin{array}{l}\text { Incremento de la cuota de mercado. } \\
\text { Incremento de la rentabilidad. } \\
\text { Incremento de la productividad. }\end{array}$ & $\begin{array}{l}\text { Valoración de la evolución de } \\
\text { los aspectos en los últimos } \\
2 \text { años }(1=\text { situación muy } \\
\text { desfavorable, } 4=\text { situación } \\
\text { muy favorable) }\end{array}$ \\
\hline $\begin{array}{l}\text { Modelo de } \\
\text { Relaciones } \\
\text { Humanas }\end{array}$ & $\begin{array}{l}\text { Aumento de la motivación de los trabajadores. } \\
\text { Reducción de la rotación de personal (abandono voluntario } \\
\text { de los trabajadores). } \\
\text { Reducción del ausentismo laboral. }\end{array}$ & $\begin{array}{l}\text { Valoración de la evolución de } \\
\text { los aspectos en los últimos } \\
2 \text { años ( } 1=\text { situación muy } \\
\text { desfavorable, } 4=\text { situación } \\
\text { muy favorable) }\end{array}$ \\
\hline
\end{tabular}

Para realizar el análisis univariante de los factores críticos de éxito, se comprobó la distribución de los datos para esto se aplicó la prueba de Shapiro-Wilk.

Posteriormente, se procedió a hacer un análisis univariante que permitió determinar cuáles de los factores identificados tienen impacto en el rendimiento competitivo de las empresas, para ello se empleó la prueba de análisis de varianza o ANOVA.

Asimismo, para validar el modelo de regresión, se efectuó la prueba de Durbin-Watson, de esta manera se comprobó la no presencia de autocorrelación de las variables del modelo, teniendo un grado de aceptación a partir del valor 2,00. Por otra parte, se efectuaron test ANOVA para confirmar la validez de la ecuación planteada a partir de un nivel de significación del 0,05 . 


\section{RESULTADOS Y DISCUSIÓN}

$\mathrm{L}$ a identificación de los posibles factores críticos de éxito fue resultado de una valoración cualitativa sobre los entornos que rodean a las empresas, y por ello, requirió de una investigación profunda y analítica enfocada hacia las dimensiones: Evaluación de la gerencias, Productor y procesos, Actividades de marketing y Tecnología e innovación. Los factores críticos preliminares encontrados se presentan en la tabla siguiente.

Tabla 2. Factores críticos de éxito en el mercado de insumos médicos en la ciudad de Sucre.

\begin{tabular}{lccc}
\hline \multicolumn{1}{c}{ Factores críticos de Éxito } & $\begin{array}{c}\text { Análisis } \\
\text { PESTEL }\end{array}$ & $\begin{array}{c}\text { Análisis } \\
\text { PORTER }\end{array}$ & $\begin{array}{c}\text { Análisis de la } \\
\text { empresa líder }\end{array}$ \\
\hline Procesos de venta (sector público y privado) & $\mathrm{X}$ & $\mathrm{X}$ & \\
Estilo de vida de los consumidores finales & $\mathrm{X}$ & $\mathrm{X}$ & $\mathrm{X}$ \\
Tecnología orientada a la venta y logística & $\mathrm{X}$ & $\mathrm{X}$ & $\mathrm{X}$ \\
Introducción de productos de mejor calidad & & $\mathrm{X}$ & $\mathrm{X}$ \\
Actividades de marketing & & $\mathrm{X}$ & $\mathrm{X}$ \\
Capacitaciones al personal & & $\mathrm{X}$ \\
Innovación en gestión de procesos & & $\mathrm{X}$ \\
Capacidad directiva & & & \\
\hline
\end{tabular}

Después de la realización de los tres análisis, se encontró congruencias respecto a 6 factores que constantemente destacaron.

En primer lugar, se consideró la posición tecnológica como un factor crítico de éxito, entendido como la estructura tecnológica en la que la empresa distribuidora apoya sus objetivos, actividades y seguimiento de procesos, tanto de venta, como de planificación y distribución de productos.

En segundo lugar, la calidad del producto, entendido como el conjunto de líneas de productos que pueden llegar a diferenciar de las líneas de productos estándar que existen en el mercado, tanto para insumos como para equipamiento o instrumental.

En tercer lugar, las capacidades del marketing, como las actividades que la empresa emplea para promocionar sus productos, y el resultado que se observa de dichas acciones.

En cuarto lugar, la innovación, desde dos perspectivas: la innovación en productos, como la capacidad de la empresa de introducir al mercado nuevos productos para diferenciarse de la competencia; y la innovación en procesos, entendido como la incorporación de nuevas técnicas, métodos orientados a mejorar la gestión de todos los procesos efectuados en la empresa y en todas las áreas posibles, ya sea para mejorar su posición en el sector público o en el sector privado. 
En quinto lugar, la formación al personal, tanto de ventas como en áreas de mantenimiento o electromedicina, entendido como el esfuerzo que realizan las empresas para procurar una constante capacitación de su personal.

Finalmente, la capacidad directiva, como la experiencia y formación del gerente de la empresa en las áreas de salud y administrativa para llevar a cabo las funciones de su cargo y la consecución de los objetivos de la empresa.

Encontrados los 6 factores críticos preliminares correspondió medir su relación con la competitividad empresarial, de esta manera se cuantificó la importancia de cada factor mediante la aplicación de un censo dirigido los responsables de las 25 empresas que conforman el sector de distribución de insumos médicos en Sucre, Bolivia, y el análisis estadístico de los datos recopilados.

Para determinar qué impacto tuvo cada uno de los factores encontrados, se procedió a dos tipos de análisis estadístico. En primer lugar, se efectuó un análisis de tipo univariante, que mide cada factor correspondiente con los indicadores de competitividad respectivo. En segundo lugar, se procedió a la realización de un análisis de regresión multivariante para explicar la incidencia de todos los factores en la competitividad de la empresa.

Los factores críticos de éxito encontrados se midieron de acuerdo con una escala de Likert, a través de la valoración de los responsables de las empresas. Por su parte, a través de la metodología de Quinn y Rorbaugh, se estableció un cuestionario para medir el nivel de competitividad de las empresas de insumos médicos a partir de 4 modelos, elaborados a través de una escala de Likert. Los indicadores son el resultado de la media aritmética de los 3 ítems de preguntas que se diseñaron para cada indicador. Finalmente, para validar la fiabilidad de la escala se procedió a realizar una prueba de Alfa de Cronbach. El criterio de aceptación para saber si la escala es confiable es a partir de 0,60. Los resultados de la prueba se muestran a continuación:

Tabla 3. Modelos de Quinn y Rorbaugh.

\begin{tabular}{llc}
\hline \multicolumn{1}{c}{ Aspectos en los que la empresa ha evolucionado } & \multicolumn{1}{c}{$\begin{array}{c}\text { Alfa de } \\
\text { Cronbach }\end{array}$} \\
\hline $\begin{array}{l}\text { Modelo de } \\
\text { Procesos Internos }\end{array}$ & $\begin{array}{l}\text { Mejora en la calidad del producto. } \\
\text { Mejora en la coordinación de procesos internos. } \\
\text { Mejor organización de las tareas del personal }\end{array}$ \\
$\begin{array}{ll}\text { Modelo de } \\
\text { Sistema abierto }\end{array}$ & $\begin{array}{l}\text { Aumento de la satisfacción de los clientes. } \\
\text { Incremento de la habilidad de adaptación a las necesidades de los } \\
\text { mercados. }\end{array}$ & \\
Mejora de la imagen de empresa y de sus productos. & \\
Modelo & $\begin{array}{l}\text { Incremento de la cuota de mercado. } \\
\text { Racional }\end{array}$ & 0,678 \\
\hline
\end{tabular}




\section{Aspectos en los que la empresa ha evolucionado}

Alfa de

Cronbach

Modelo de

Relaciones Humanas

Aumento de la motivación de los trabajadores.

0,621

Reducción de la rotación de personal (abandono voluntario de los

trabajadores).

Reducción del ausentismo laboral.

Como la prueba de Alfa de Cronbach arroja resultados por encima del 0,60 , se valida la escala y se procede a la interpretación de los resultados obtenidos

Tabla 4. Grado de competitividad en empresas distribuidores de insumos médicos.

\begin{tabular}{lcccccc}
\hline \multicolumn{1}{c}{ Ítem } & $\begin{array}{c}\text { Situación } \\
\text { muy } \\
\text { desfavorable }\end{array}$ & $\begin{array}{c}\text { Situación } \\
\text { bastante } \\
\text { desfavorable }\end{array}$ & $\begin{array}{c}\text { Situación } \\
\text { favorable }\end{array}$ & $\begin{array}{c}\text { Situación } \\
\text { bastante } \\
\text { favorable }\end{array}$ & $\begin{array}{c}\text { Situación } \\
\text { muy } \\
\text { favorable }\end{array}$ & Total \\
\hline $\begin{array}{l}\text { Mejora en la calidad de } \\
\text { productos }\end{array}$ & 16.00 & 28.00 & 32.00 & 20.00 & 4.00 & 100.00 \\
$\begin{array}{l}\text { Coordinación de } \\
\text { procesos }\end{array}$ & 12.00 & 4.00 & 40.00 & 36.00 & 8.00 & 100.00 \\
$\begin{array}{l}\text { Organización de tareas } \\
\text { Satisfacción de clientes }\end{array}$ & 12.00 & 36.00 & 16.00 & 28.00 & 8.00 & 100.00 \\
$\begin{array}{l}\text { Adaptación al mercado } \\
\begin{array}{l}\text { Mejora de la imagen y } \\
\text { productos }\end{array}\end{array}$ & 12.00 & 12.00 & 52.00 & 32.00 & 4.00 & 100.00 \\
$\begin{array}{l}\text { Incremento de la cuota } \\
\text { de mercado }\end{array}$ & 12.00 & 32.00 & 40.00 & 16.00 & 0.00 & 100.00 \\
$\begin{array}{l}\text { Incremento de la } \\
\text { rentabilidad }\end{array}$ & 4.00 & 24.00 & 24.00 & 28.00 & 12.00 & 100.00 \\
$\begin{array}{l}\text { Incremento de la } \\
\text { productividad }\end{array}$ & 4.00 & 36.00 & 28.00 & 28.00 & 12.00 & 100.00 \\
$\begin{array}{l}\text { Motivación del } \\
\text { personal }\end{array}$ & 12.00 & 28.00 & 32.00 & 24.00 & 4.00 & 100.00 \\
$\begin{array}{l}\text { Rotación del personal } \\
\begin{array}{l}\text { Ausentismo del } \\
\text { personal }\end{array}\end{array}$ & 0.00 & 0.00 & 20.00 & 48.00 & 32.00 & 100.00 \\
\hline & 0.00 & 0.00 & 20.00 & 48.00 & 32.00 & 100.00 \\
\hline
\end{tabular}


Como se indicó anteriormente, la formulación de los indicadores de competencia en los cuatro modelos de Quinn y Rorbaugh, resultan de la media aritmética de los 3 ítems por cada modelo, de esta forma se formularon los 4 indicadores correspondientes a los cuatro modelos, incluyendo un resultado global, resultado de la media aritmética de los 12 ítems. De esta forma, como se aprecia en la tabla presentada se logró calcular los indicadores para todas las empresas, asimismo existe una gran diferencia entre los mínimos y máximo valores de las empresas, esto demuestra que existe una gran diferencia entre la empresa menos competitiva del mercado y la empresa más competitiva de acuerdo con cada modelo.

Se realizó un análisis univariante de los factores críticos de éxito, se comprobó la distribución de los datos, para corroborar si los mismos responden a una distribución normal. Para ello, se empleó la prueba de Shapiro-Wilk que se expresa de la siguiente forma:

Ho: La distribución de los datos es normal

Ha: La distribución de los datos no es normal

Nivel de significancia: 0,05

Tabla 5. Resultados de Shapiro-Wilk para pruebas de normalidad.

\begin{tabular}{|c|c|c|c|}
\hline \multirow{2}{*}{ Ítem } & \multicolumn{3}{|c|}{ Shapiro-Wilk } \\
\hline & Estadístico & gl & Sig. \\
\hline Capacidad Directiva & 870 & 25 &, $064^{\star}$ \\
\hline Posición tecnológica & ,854 & 25 & $102^{*}$ \\
\hline Calidad de productos & ,850 & 25 &, $082^{\star}$ \\
\hline Capacidad del marketing & 902 & 25 & $200^{*}$ \\
\hline Formación del personal & 610 & 25 &, $070^{\star}$ \\
\hline Innovación de productos & 915 & 25 & $640^{*}$ \\
\hline Innovación de procesos & ,881 & 25 &, $577^{\star}$ \\
\hline Modelo de Procesos Internos & 967 & 25 &, $582^{*}$ \\
\hline Modelo de Sistema Abierto & 969 & 25 & $625^{\star}$ \\
\hline Modelo Racional & 953 & 25 & $300^{*}$ \\
\hline Modelo de Recursos Humanos & ,882 & 25 &, $078^{\star}$ \\
\hline Resultado global & 919 & 25 &, $069^{\star}$ \\
\hline
\end{tabular}

$\left(^{\star}\right)$ Para sig.> 0,05 
Dado que todos los valores de significancia para las variables sujetas a las pruebas de normalidad, presentan valores que se encuentran encima del nivel de significancia de 0,05, se aceptó la hipótesis nula y se rechazó la hipótesis alterna, por lo que se comprueba que los datos responden a una distribución normal.

\section{Formulación de hipótesis}

Como se mencionó anteriormente, se procedió a hacer un análisis univariante para identificar si efectivamente los factores identificados tienen impacto en el rendimiento competitivo de las empresas, para ello se empleó la prueba de Análisis de varianza o ANOVA. Las hipótesis planteadas son las siguientes:

- HO: La posición tecnológica no tiene un efecto positivo en la competitividad de las empresas distribuidoras de insumos médicos

- H1: La posición tecnológica tiene un efecto positivo en la competitividad de las empresas distribuidoras de insumos médicos.

- HO: La calidad de los productos no tiene un efecto positivo en la competitividad de las empresas distribuidoras de insumos médicos

- H2: La calidad de los productos tiene un efecto positivo en la competitividad de las empresas distribuidoras de insumos médicos.

- HO: La innovación de los productos no tiene un efecto positivo en la competitividad de las empresas distribuidoras de insumos médicos

- H3: La innovación de los productos tiene un efecto positivo en la competitividad de las empresas distribuidoras de insumos médicos.

- HO: La innovación en procesos no tiene un efecto positivo en la competitividad de las empresas distribuidoras de insumos médicos

- H4: La innovación en procesos tiene un efecto positivo en la competitividad de las empresas distribuidoras de insumos médicos.

- HO: La formación al personal no tiene un efecto positivo en la competitividad de las empresas distribuidoras de insumos médicos

- H5: La formación al personal tiene un efecto positivo en la competitividad de las empresas distribuidoras de insumos médicos.

- HO: Las capacidades del marketing no tiene un efecto positivo en la competitividad de las empresas distribuidoras de insumos médicos

- H6: Las capacidades del marketing tiene un efecto positivo en la competitividad de las empresas distribuidoras de insumos médicos.

- HO: La capacidad directiva no tiene un efecto positivo en la competitividad de las empresas distribuidoras de insumos médicos

- H7: La capacidad directiva tiene un efecto positivo en la competitividad de las empresas distribuidoras de insumos médicos. 
Tabla 6. Resultados del análisis univariante.

\begin{tabular}{|c|c|c|c|c|c|c|}
\hline \multicolumn{7}{|c|}{ Modelos } \\
\hline FCE & & $\begin{array}{l}\text { Procesos } \\
\text { internos }\end{array}$ & $\begin{array}{l}\text { Sistema } \\
\text { Abierto }\end{array}$ & Racional & $\begin{array}{l}\text { Recursos } \\
\text { Humanos }\end{array}$ & $\begin{array}{c}\text { Resultado } \\
\text { Global }\end{array}$ \\
\hline \multirow{2}{*}{ Posición tecnológica } & Beta & 0,680 & 0,555 & 0,627 & 0,433 & 0,654 \\
\hline & Sig. & $0,000^{\star \star}$ & $0,004^{\star \star}$ & $0,001^{\star \star}$ & $0,08^{\star}$ & $0,000^{\star *}$ \\
\hline \multirow{2}{*}{ Calidad de productos } & Beta & 0,722 & 0,678 & 0,702 & 0,545 & 0,748 \\
\hline & Sig. & $0,000^{\star \star}$ & $0,000^{\star \star}$ & $0,000^{\star \star}$ & $0,005^{\star \star}$ & $0,000^{\star \star}$ \\
\hline \multirow{2}{*}{ Innovación de productos } & Beta & 0,248 & 0,584 & 0,412 & 0,358 & 0,506 \\
\hline & Sig. & $0,059^{\star}$ & $0,002^{\star \star}$ & $0,041^{\star \star}$ & $0,079^{\star}$ & $0,010^{\star \star}$ \\
\hline \multirow{2}{*}{ Innovación de procesos } & Beta & 0,689 & 0,717 & 0,691 & 0,441 & 0,722 \\
\hline & Sig. & $0,000^{\star \star}$ & $0,000^{\star \star}$ & $0,000^{\star \star}$ & $0,027^{\star \star}$ & $0,000^{\star \star}$ \\
\hline \multirow{2}{*}{ Formación del personal } & Beta & 0,364 & 0,421 & 0,447 & 0,147 & 0,395 \\
\hline & Sig. & $0,074^{\star}$ & $0,036^{\star \star}$ & $0,025^{\star \star}$ & $0,084^{\star}$ & $0,052^{\star}$ \\
\hline \multirow{2}{*}{ Capacidad del marketing } & Beta & 0,824 & 0,796 & 0,676 & 0,779 & 0,863 \\
\hline & Sig. & $0,000^{\star \star}$ & $0,000^{\star \star}$ & $0,000^{\star \star}$ & $0,000^{\star \star}$ & $0,000^{\star \star}$ \\
\hline \multirow{2}{*}{ Capacidad Directiva } & Beta & 0,635 & 0,765 & 0,597 & 0,586 & 0,725 \\
\hline & Sig. & $0,001^{\star \star}$ & $0,000^{\star \star}$ & $0,002^{\star \star}$ & $0,002^{\star \star}$ & $0,000^{\star *}$ \\
\hline
\end{tabular}

Test ANOVA c/ nivel de significación 0,05

(*) Para sig.> 0,05 // (**) Para sig.<0,05

Después de realizar el análisis univariante, los resultados nos indican que:

- La posición tecnológica, resulta no ser un factor con efecto positivo en la competitividad en el modelo de Recursos Humanos, es un factor positivo en los demás modelos, teniendo mayor efecto en el modelo de Procesos Internos $(B=0,680)$, de esta forma, la H1 se acepta en todos los modelos, exceptuando Recursos Humanos.

- La calidad de productos, es un factor con efecto positivo en todos los modelos propuestos, teniendo mayor efecto en el modelo del Resultado Global $(B=0,748)$, por tanto, la $\mathrm{H} 2$ se acepta en todos los modelos

- La innovación de productos, resulta ser un factor con efecto en los modelos de Sistema Abierto, Racional y Resultado global, no teniendo así un efecto en los modelos de Procesos Internos y Recursos Humanos, por tanto, la H3 queda rechazada.

- La innovación en procesos, tiene un efecto positivo en todos los modelos propuestos, teniendo mayor impacto en el modelo de Resultado Global con $(B=0,722)$. Es así, que la H4 se acepta para todos los modelos. 
- La formación del personal, no representa un efecto para los modelos de Procesos Internos, Recursos Humanos y Resultado global, solo teniendo un efecto positivo en los modelos de Sistema Abierto y el modelo Racional, con mayor efecto en el modelo Racional. Considerando que la $\mathrm{H} 5$ no se acepta en 3 de los 5 modelos, la misma es rechazada.

- Las capacidades del marketing, tienen un efecto positivo en la competitividad de acuerdo a todos los modelos propuestos, teniendo mayor efecto en el modelo de Resultado global, por ello la H6 se acepta.

- La capacidad directiva, se constituye como un factor con efecto positivo en todos los modelos, teniendo mayor impacto en el modelo de Sistema Abierto $(B=0,765)$, por tanto, la $\mathrm{H} 7$ se acepta en todos los modelos.

Después de realizar el análisis univariante, se demostró que los factores de innovación de productos y formación al personal no resultaron relevantes en la competitividad de las distribuidoras de insumos médicos.

\section{Análisis multivariante de los factores críticos de éxito}

Para el análisis multivariante, se consideró únicamente las hipótesis que resultaron aceptadas en el análisis univariante. Para validar el modelo de regresión para los 5 indicadores, se han efectuado la prueba de Durbin-Watson para validar la no presencia de autocorrelación de las variables que se estudian en dicho modelo, teniendo un grado de aceptación a partir del valor 2,00. Por otra parte, se efectuaron test ANOVA para efectuar la validez de la ecuación planteada a partir de un nivel de significación del 0,05, Rubio (2012).

Tabla 7. Resultados de test Durbin-Watson/ ANOVA.

\begin{tabular}{llllllllll}
\hline $\begin{array}{c}\text { Modelo de } \\
\text { procesos internos }\end{array}$ & $\begin{array}{c}\text { Modelo de Sistema } \\
\text { Abierto }\end{array}$ & Modelo Racional & $\begin{array}{c}\text { Modelo de } \\
\text { Recursos humanos }\end{array}$ & Resultado Global \\
\hline $\begin{array}{l}\text { Durbin- } \\
\text { Watson }\end{array}$ & ANOVA & $\begin{array}{l}\text { Durbin- } \\
\text { Watson }\end{array}$ & ANOVA & $\begin{array}{l}\text { Durbin- } \\
\text { Watson }\end{array}$ & ANOVA & $\begin{array}{c}\text { Durbin- } \\
\text { Watson }\end{array}$ & ANOVA & $\begin{array}{c}\text { Durbin- } \\
\text { Watson }\end{array}$ & ANOVA \\
\hline 1,990 & $000 \mathrm{~b}$ & 1,913 &, $000 \mathrm{~b}$ & 1,816 &, $000 \mathrm{~b}$ & 1,807 &, $001 \mathrm{~b}$ & 1,361 &, $000 \mathrm{~b}$ \\
\hline
\end{tabular}

Teniendo estos resultados se ha podido comprobar la validez del estudio de regresión, pudiendo entonces también validar los resultados que aparecen en el modelo. 
Tabla 8. Resultados del análisis multivariante.

\begin{tabular}{|c|c|c|c|c|c|c|c|c|c|c|}
\hline \multirow{2}{*}{\begin{tabular}{l}
\multicolumn{1}{c}{ FCE } \\
$\begin{array}{l}\text { Capacidad } \\
\text { Directiva }\end{array}$
\end{tabular}} & \multicolumn{2}{|c|}{$\begin{array}{l}\text { Modelo de } \\
\text { procesos } \\
\text { internos }\end{array}$} & \multicolumn{2}{|c|}{$\begin{array}{c}\text { Modelo de } \\
\text { Sistema Abierto }\end{array}$} & \multicolumn{2}{|c|}{$\begin{array}{l}\text { Modelo } \\
\text { Racional }\end{array}$} & \multicolumn{2}{|c|}{$\begin{array}{c}\text { Modelo de } \\
\text { Recursos } \\
\text { humanos }\end{array}$} & \multicolumn{2}{|c|}{$\begin{array}{c}\text { Resultado } \\
\text { Global }\end{array}$} \\
\hline & Beta & 0,071 & Beta & 0,318 & Beta & 0,098 & Beta & 0,196 & Beta & 0,185 \\
\hline & Sig. & 0,553 & Sig. & 0,023 & Sig. & 0,563 & Sig. & 0,326 & Sig. & $0,043^{\star}$ \\
\hline \multirow{2}{*}{$\begin{array}{l}\text { Posición } \\
\text { tecnológica }\end{array}$} & Beta & 0,306 & Beta & 0,171 & Beta & 0,285 & Beta & 0,082 & Beta & 0,247 \\
\hline & Sig. & $0,008^{\star}$ & Sig. & 0,142 & Sig. & $0,045^{\star}$ & Sig. & 0,635 & Sig. & $0,004^{\star}$ \\
\hline \multirow[t]{2}{*}{$\begin{array}{l}\text { Calidad de } \\
\text { productos }\end{array}$} & Beta & 0,096 & Beta & 0,049 & Beta & 0,198 & Beta & 0,016 & Beta & 0,103 \\
\hline & Sig. & 0,447 & Sig. & 0,717 & Sig. & 0,272 & Sig. & 0,939 & Sig. & 0,265 \\
\hline \multirow[t]{2}{*}{$\begin{array}{l}\text { Capacidad del } \\
\text { marketing }\end{array}$} & Beta & 0,444 & Beta & 0,364 & Beta & 0,190 & Beta & 0,633 & Beta & 0,449 \\
\hline & Sig. & $0,002^{\star}$ & Sig. & $0,014^{\star}$ & Sig. & 0,289 & Sig. & $0,006^{\star}$ & Sig. & $0,000^{*}$ \\
\hline \multirow[t]{2}{*}{$\begin{array}{l}\text { Innovación de } \\
\text { procesos }\end{array}$} & Beta & 0,255 & Beta & 0,250 & Beta & 0,323 & Beta & $-0,039$ & Beta & 0,235 \\
\hline & Sig. & $0,036^{*}$ & Sig. & $0,048^{\star}$ & Sig. & 0,058 & Sig. & 0,834 & Sig. & $0,010^{\star}$ \\
\hline
\end{tabular}

Nivel de significación $=0,05$

$\left({ }^{\star}\right)$ Sig. $<0,05$

En la tabla anterior, indica que conforme al análisis multivariante existe un efecto positivo del indicador Capacidad Directiva, únicamente en el modelo de Resultado global, por otra parte, el indicador de posición tecnológica resulta manifestar un efecto positivo en el modelo de procesos internos, modelo racional y resultado global, mostrando mayor efecto.

Así mismo, se puede observar un efecto positivo en la competitividad de las distribuidoras de insumos médicos a partir de la capacidad del marketing, pues es un factor que resulta en dicho efecto en el modelo de procesos internos, modelo de sistema abierto, de recursos humanos y resultado global, no mostrando un efecto únicamente en el modelo Racional.

Por otro lado, la variable innovación en procesos muestra un efecto positivo en el modelo de procesos internos, modelo de sistema abierto y resultado global.

Finalmente, la variable calidad de productos resulta no tener ningún efecto positivo en ninguno de los modelos propuestos, por tanto, se descartó como un factor crítico de éxito de las empresas distribuidoras de insumos médicos en la ciudad de Sucre. 


\section{CONCLUSIONES}

$\mathrm{E}$ análisis científico y riguroso de factores críticos de éxito perite al sector de insumos médicos en la ciudad de Sucre elevar la competitividad, debido a que permite destinar esfuerzos y recursos a aquellos eventos, condiciones, circunstancias o actividades que generan efecto positivo en todas o la mayoría de las áreas de la empresa.

La primera etapa del estudio, con base en herramientas de alcance descriptivo sugirió seis factores estratégicos que constantemente destacaron: La posición tecnológica, La calidad de productos, La innovación de productos, La innovación en procesos, La formación del personal y La capacidad directiva.

En la segunda etapa del estudio, mediante un análisis univariado se descartaron dos de estos factores por no tener significancia estadística en la competitividad empresarial (innovación de productos y formación al personal).

Realizadas las pruebas estadísticas y conforme al análisis multivariante se provee de un análisis mucho más específico y se afirma que existe un efecto positivo del indicador Capacidad Directiva, únicamente en el modelo de Resultado global, por otra parte, el indicador de posición tecnológica resulta manifestar un efecto positivo en el modelo de procesos internos, modelo racional y resultado global, mostrando mayor efecto.

Así mismo, se puede observar un efecto positivo en la competitividad de las distribuidoras de insumos médicos a partir de la capacidad del marketing, pues es un factor que resulta en dicho efecto en el modelo de procesos internos, modelo de sistema abierto, de recursos humanos y resultado global, no mostrando un efecto únicamente en el modelo Racional.

Por otro lado, la variable innovación en procesos muestra un efecto positivo en el modelo de procesos internos, modelo de sistema abierto y resultado global.

Finalmente, la variable calidad de productos resulta no tener ningún efecto positivo en ninguno de los modelos propuestos, por tanto, se descarta como un factor crítico de éxito de las empresas distribuidoras de insumos médicos en la ciudad de Sucre.

Con base en los resultados de este estudio, se recomienda elaborar un modelo de gestión por factores críticos de éxito aplicable a empresas de este rubro, ya que de esta manera estas instituciones podrán plantearse acciones orientadas a las actividades clave, de manera más eficiente y con procesos de mayor calidad.

\section{REFERENCIAS}

Aragón Sánchez, A., y Rubio Bañón, A. (2005). Factores asociados con el éxito competitivo de las pyme industriales en España. Universia Business Review, núm. 8, cuarto trimestre, pp. 38-51 Portal Universia S.A. Madrid, España

Estrada, R; García, D, y Sánchez, V. (2009). Factores determinantes del éxito competitivo en la Pyme: Estudio Empírico en México. Revista venezolana de Gerencia, vol. 14, núm. 46, abril-junio

King, S., y Burgess, F. (2006). Beyond critical success factors: A dynamic model of enterprise system innovation. International Journal of information management, 26(1), 59-69 
Mendoza, E.; Marius, A.; Pérez, M., y Grimán, C. (2007). Critical success factors for a customer relationship management strategy. Information and software technology, 49(8), 913-945

PROCHILE. (2016). Perfil de mercado de equipos e insumos médicos Bolivia. Septiembre 2016. P. 25

Romero, R.; Morales, S.; Toledo, C., y Delgado, V. (2009). Factores críticos de éxito: Una estrategia de competitividad. CULCYT: Cultura Científica y Tecnológica. ISSN 2007-0411, N. 31, pags. 5-14.
Rubio, M., y Berlanga, V. (2012). Cómo aplicar las pruebas paramétricas bivariadas t de Student y ANOVA en SPSS. Caso práctico. [En línea] REIRE, Revista d'Innovació i Recerca en Educació, Vol. 5, núm. 2, 83100. Accesible en: http://www.ub.edu/ ice/reire.htm

Villegas, G. (2005). Factores Críticos de éxito. REVISTA EAFIT - 105. Colombia 